ential equations of elliptic type in two independent variables. The kernels of these operators can be expressed in terms of the Riemann function, dependent on the coefficients in the partial differential equation. As an illustration, the connexion of special cases with the Bessel and Legendre functions is shown. The imposition of boundary value conditions effects a reduction to integral equations: these may be of Fredholm type, but much use is made of singular integral equations involving principal value integrals, a field in which Muskhelishvili's 1946 book (with an English translation in 1953) is a basic reference. Applications are made to vibrations of a membrane, elastic spherical shells and other elasticity problems.

The reader should possess a sound knowledge of the classical theory of functions of a complex variable, and of the basic equations of theoretical elasticity. He will then appreciate the book as another example of the way in which Soviet mathematicians exploit the resources of high-powered analysis in the practical application of mathematical theories. $\quad$ T. A. A. BROADBENT

\section{OPERATIONAL CALCULUS}

Introduction to the Operational Calculus

By L. Berg. English translation of the second edition. (North-Holland Series on Applied Mathematics and Mechanies, Vol. 2.) Pp. $x+294$. (Amsterdam: NorthHolland Publishing Company, 1967.) $80 s$.

Operational calculus was developed by Heaviside and used by many of his successors on an intuitive basis. The closest approach to a mathematical theory of the original Heaviside calculus is presented by J.P. Dalton in Symbolic Operators in 1954. About fifty years ago it was discovered that Laplace integrals could be used effectively as a substitute for operational calculus, and since then most investigators aiming at precision and mathematical rigour have used Laplace transforms.

Laplace transforms, however, have some shortcomings from the point of view of an applied mathematician. Laplace integrals do not have any obvious physical significance; in order to ensure their convergence, it is necessary to impose certain unnatural growth restrictions on the functions subjected to the transformation; and Laplace transforms involve the values of the functions concerned for all positive values of the variable, while the physical processes to which they are applied depend at any time $t$ on the past of the system, but not on its future.

About 1950, Jan Mikusinski developed an operational calculus which is free from these blemishes and is mathematically rigorous. Mikusinski's operational calculus is based on the convolution integral

$$
\int_{0}^{t} f(u) g(t-u) \mathrm{d} u
$$

which is Duhamel's integral and can serve to introduce a species of product in the space of continuous functions of a positive variable. Continuous functions endowed with pointwise addition and convolution as multiplication form an algebraic system to which the usual processes of modern abstract algebra can be applied, and lead not only to an operational calculus but also to a concept of generalized functions including impulse functions. The function which is constant and equal to unity serves as the operator of integration, its reciprocal is Heaviside's operator of differentiation, the unit element of the algebraic system constructed by Mikusinski is the delta function, and so on.

The book being reviewed has grown out of courses of lectures delivered by Berg to students of mathematies, physics and electrical engineering. The author presents both the Laplace transform approach and Mikusinski's operational calculus, and he describes more briefly an extension of Laplace transforms which relaxes considerably the global integrability conditions. The reader is assumed to be thoroughly familiar with advanced calculus and the elements of complex variable theory, but in other respects the book is self-contained, and includes, for example, a detailed exposition of the concepts and methods of abstract algebra needed in the sequel. Before developing Mikusinski's operational calculus, Berg presents the corresponding operational calculus for functions of a discrete variable, that is, for sequences. This is in the nature of a preliminary exercise, and serves to introduce some of the concepts and techniques that will appear in later parts of the book, but the calculus so developed is of interest for its own sake. In the development of operational calculus for functions of a continuous variable, the author departs from Mikusinski in that he introduces the modified convolution product

$$
f g(t)=\frac{\mathrm{d}}{\mathrm{d} t} \int_{0}^{t} f(u) g(t-u) \mathrm{d} u
$$

in order to achieve certain formal simplifications. The operational calculus so developed is then applied to the solution of ordinary differential equations with constant coefficients, systems of such equations, and to integral equations of the convolution type.

Laplace transforms are also developed and applied to the solution of ordinary linear differential equations with polynomial coefficients, partial differential equations, difference equations, and integral equations of convolution type. Additional material includes asymptotics; an extended concept of Laplace transforms; an operational calculus based on the finite Laplace transformation; operational calculus for functions of two variables; and an operational calculus based on Volterra's theory of compositions and applicable to ordinary linear differential equations with variable coefficients.

The first German edition of this book appeared in 1961, and sold so well that a second corrected and somewhat enlarged edition could be published by 1964. The translation reviewed here is based on this second edition: problems with answers have been added. A. ERDíLYr

\section{DIFFICULT FLUIDS}

\section{Non-Newtonian Flow and Heat Transfer}

By A. H. P. Skelland. Pp. xvi +469. (New York and London: John Wiley and Sons, Inc., 1967.) $140 s$.

THE time is undoubtedly ripe for a general book on nonNewtonian technology. A few years ago the subject was sufficiently new and specialized for original papers to be the appropriate source of basic information, but the body of knowledge and the range of users have grown so far that a readable guide to the state of knowledge is very welcome. With the exception of Wilkinson's monograph, Non-Newtonian Fluids, this field has been noticeably lacking in such surveys, and Professor Skelland has done a good service in undertaking the task. The size of the task is perhaps indicated by the length of the bibliographies attached to each chapter. More than four hundred books and papers are quoted, so that this book would provide an excellent start to a literature search.

The author has aimed to make his book useful to a wide range of readers, while keeping engineering application, rather than analytical detail, to the forefront. The practising engineer who meets an isolated problem in non-Newtonian flow will find here all the information he needs, if it exists yet, although it is not always presented in the most convenient form for immediate use. The undergraduate will appreciate the down to earth approach and the worked and unworked examples, but may be 